\title{
A novel phase shift technique in shearography for NDT
}

\author{
Zhanwei Liu*, Jianxin Gao \\ TWI Ltd, Granta Park, Great Abington, Cambridge CB21 6AL,UK
}

\section{Abstract:}

Digital shearography (DS) is a whole-field non-contacting optical method for nondestructive testing (NDT) of subsurface flaws and strain measurement. In this paper, we describe a novel phaseshifting technique in DS for nondestructive evaluation (NDE) and NDT of quasi dynamic behaviour of objects subject to varying loads. A Wollaston lens, a precise wave plate device and a polariser are employed to introduce constant phase differences between two shearing wavefronts. The wave plate device is in front of the polariser, which has a transmissive wavefront distortion of less than 1/8 wavelength. Phase difference can be adjusted by rotating the polariser. The rotation for angle adjusting can be easily carried out according to the requirement of the phase shifting. In this technique, the phase shifting is performed only on the static images of the object before deformation or loading. By setting the angle of the polariser to $0^{\circ},-60^{\circ}$ and $60^{\circ}$, three speckle patterns with the defined phase differences are captured, and are referred to as initial speckle patterns I1,0, I1,-60 and I1,60. Afterwards, the polariser is reset to $0^{\circ}$ before loading. The speckle pattern I 0 is treated as a base pattern, and will be subtracted from the subsequent speckle patterns which are captured in the quasi dynamic deformation process.

During the quasi dynamic deformation process, the optical path and the entire optical set-up will be kept unchanged, and a series of speckle patterns $\left(\mathrm{I}_{\mathrm{m}, 0}, \mathrm{~m}=2,3, \ldots ..\right)$ which carry the deformation information will be quickly captured. In the meantime, fast image subtractions will be performed. The speckle fringes $\left(I_{m, 0}-I_{1,0}\right)$ will be displayed in real-time. In detailed post-processing, phase of differences method (PDM) [1] is used to extract phase information from the speckle images Im,0 - $\mathrm{I}_{1,0}$, $\mathrm{I}_{1,0}, \mathrm{I}_{1,-60}$ and $\mathrm{I}_{1,60}$. Specifically, when a three phase step technique is employed, the phase of the fringe pattern $(I m, 0-I 1,0)$ can be calculated from $\left(I_{m, 0}-I_{1,0}\right)+I_{1,0}-I_{1,-60},\left(I_{m, 0}-I_{1,0}\right)$, and $\left(I_{m, 0}-I_{1,0}\right)+I_{1,0}-I_{1,60}$. Because this temporal phase shifting is not performed in the deformation process, the technique is suitable for NDT and NDE of quasi dynamic deformation behaviour of an object.

The technique only requires three speckle patterns with phase differences prior to the object deformation. When a varying or quasi dynamic load is applied on the object, no physical phase shifting is needed to be conducted when sequential speckle patterns are recorded by a digital camera during the deformation process. Compared with the $(5, n)$ temporal phase shifting techniques [2] using a PZT driver, the proposed technique has three main advantages. Firstly, it does not need a tedious calibration process for phase shifting. Secondly, the optical set-up is relatively simple. Thirdly, the speckle fringe patterns which will reflect the underlying defect can be displayed in realtime. Compared with the spatial phase shifting techniques using a phase mask $[3,4]$ which are at the sacrifice of a weakened light intensity, the current technique is a light intensity loss-free method.

*e-mail : zhanwei.liu@twi.co.uk 
Compared with conventional polarisation phase shifting techniques [5], it does not need a complicated light path splitter or an expensive split grating, nor does a micro polarizer array. As an application of the proposed technique, a non-contacting inspection of an impact defect in a composite panel and its mechanical behaviour under different loading conditions is provided. During the experiment, three phase shifted images were recorded before thermal loading was applied.

Subsequent image series during thermal stressing were recorded to analyse the continuous deformation behaviour over time. Experimental results are shown in Fig.1 and Fig.2, where Fig.1 depicts the unwrapped phase pattern, while Fig. 2 shows the derivative of W (out-of-plane displacement) with respect to $\mathrm{x}$ axis as of Fig.1. This experiment demonstrated the feasibility of the technique.

It should be noted that, when deformation is too large, de-correlation will occur, which will result in poor visibility of the fringe patterns $\left(I_{n, 0}-I_{1,0}\right)$, or the fringe patterns will disappear completely. This problem usually happens in a quasi dynamic test, and was not resolved in [2]. Our future work will focus on calculating the phase of speckle fringes $\left(I_{n, 0} .-I_{m 0}\right.$, here $\left.n>m>1\right)$ by reconstructing new speckle pattern $I_{m,-60}$ and $I_{m, 60}$ using only the fringe $\left(I_{m, 0}-I_{1,0}\right)$ and the initial phase shifting speckle patterns $\mathrm{I}_{1,0}, \mathrm{I}_{1,-60}$ and $\mathrm{I}_{1,60}$. This will be based on the analysis of the relationships between the intensity, the phase and the amplitude of the speckle and fringes patterns.

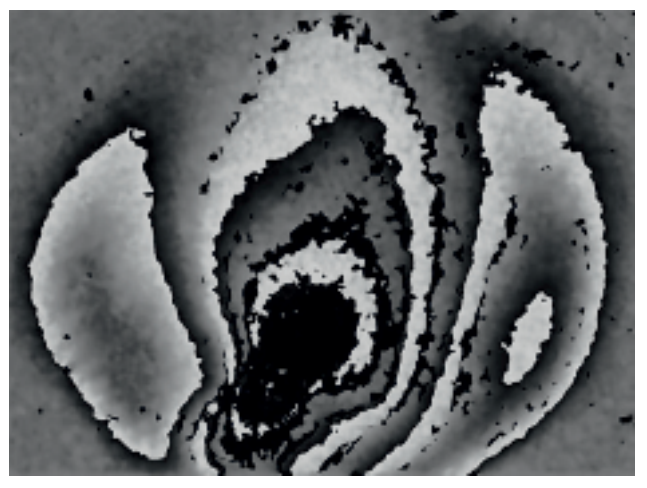

Figure 1 Unwrapped phase after filtering.

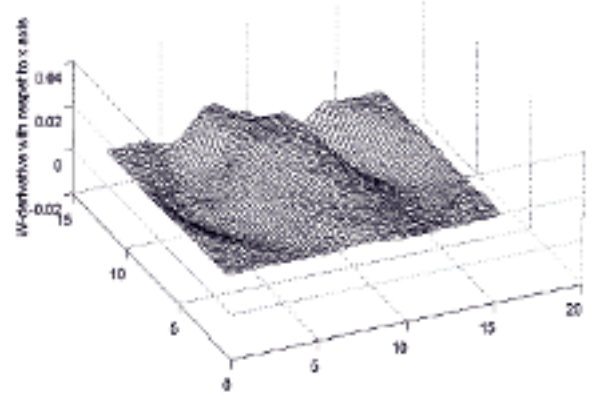

Figure 2 Derivative of $\mathrm{W}$ with respect to $\mathrm{x}$ axis (horizontal direction)

\section{References:}

[1] B. Bhaduri, M.P. Kothiyal,N.K. Mohan, Optik, 2008

[2] B. Bhaduri, N.K. Mohan, M.P. Kothiyal, J. Holo. Speckle, 2006

[3] B. Bhaduri, N.K. Mohan, M.P. Kothiyal, Optics Express, 2006

[4] B. Bhaduri, N.K. Mohan, M.P. Kothiyal, Optical Engineering, 2007

[5] Y J Yu, J Peng1 and Z Q Wang, Journal of Physics: Conference Series, 2006 